\title{
Assessment of cavopulmonary connections by advanced imaging: value of flat-detector computed tomography
}

\author{
Martin Glöckler, ${ }^{1}$ Andreas Koch, ${ }^{1}$ Julia Halbfaß, ${ }^{1}$ Verena Greim, ${ }^{1}$ Andrè Rüffer, ${ }^{2}$ Robert Cesnjevar, ${ }^{2}$ \\ Stephan Achenbach, ${ }^{3}$ Sven Dittrich ${ }^{1}$ \\ ${ }^{1}$ Department of Pediatric Cardiology; ${ }^{2}$ Department of Congenital Heart Surgery; ${ }^{3}$ Department of Cardiology, \\ University Hospital Erlangen, Erlangen, Germany
}

\begin{abstract}
Objectives: To investigate the impact of flat-detector computed tomography on the clinical assessment of patients with cavopulmonary connections, and to evaluate the obtained diagnostic accuracy and supplementary information, as well as the value of overlaid three-dimensional reconstructions on fluoroscopic images during catheter-based interventions. Methods: We analysed 31 consecutive patients retrospectively in whom flat-detector computed tomography was used to visualise the cavopulmonary connection. We investigated patients with cavopulmonary connections either early post-operatively (first group), before converting to a total cavopulmonary connection (second group), and patients with failing total cavopulmonary connection (third group). Flat-detector computed tomography based on a single rotational angiography was used to create a three-dimensional vascular model. The clinical value of flat-detector computed tomography was evaluated using standard categories of diagnostic utility. Used contrast volume and radiation exposure were quantified. Results: Within 18 months, flat-detector computed tomography was performed in 31 cases with cavopulmonary connections. The median age was 1.9 years (range $0.3-43$ years). In the first group, we found anomalies in 4 out of 8 cases, which led to therapeutic or prophylactic procedures; in the second and third groups, we performed interventions in 14 out of 23 cases. The overall clinical value was always rated superior to conventional biplane angiography. The median dose area product was 91.8 microgray square metres (range 33.0-679.3 microgray square metres). The required contrast medium was 2.08 millilitres per kilogram (range 0.66-4.7 millilitres per kilogram). Conclusion: Flat-detector computed tomography improves the diagnostic accuracy in cavopulmonary connections and provides additional diagnostic information, which may lead to therapeutic or prophylactic procedures. Overlaid three-dimensional images on fluoroscopy facilitate and provide security for interventions.
\end{abstract}

Keywords: Three-dimensional; rotational angiography; interventions in congenital heart disease

Received: 7 September 2011; Accepted: 5 February 2012; First published online: 8 March 2012

A $\mathrm{N}$ UNRESTRICTED PULMONARY BLOOD FLOW IS crucial in cavopulmonary connections. ${ }^{1-3}$ Consequently, there is a significant need for sufficient image information. The echocardiographic window in this region is often limited and frequently

Correspondence to: Dr M. Glöckler, MD, Department of Pediatric Cardiology, University Hospital Erlangen, Loschgestrasse 15, D-91054 Erlangen, Germany. Tel: +499131 8533750; Fax: +4991318535987; E-mail: martin.gloeckler@ uk-erlangen.de inadequate; ${ }^{4}$ therefore, another imaging modality has to be added whenever diagnostic imaging is required. In clinical routine, cardiac catheterisation, in addition to magnetic resonance imaging and multi-detector row computed tomography, is an important imaging modality in cavopulmonary connections. A routine cardiac catheterisation before partial cavopulmonary connection seems to be avoidable in most cases, ${ }^{5}$ but after surgically performed partial cavopulmonary connection unexpected pathological findings are 
Table 1. Demographic and procedural data, median (with range), number (percentage of total).

\begin{tabular}{lcccc}
\hline Parameter & All & First group & Second group & Third group \\
\hline Number & $31(100)$ & $8(26)$ & $13(42)$ & $10(32)$ \\
Gender $(\mathrm{m} / \mathrm{f})$ & $19 / 12$ & $4 / 4$ & $11 / 2$ & $4 / 6$ \\
Age $($ years $)$ & $1.9(0.3-42.5)$ & $0.4(0.3-7.3)$ & $1.7(0.5-42.5)$ & $8.6(2.0-16.3)$ \\
Height $(\mathrm{cm})$ & $87(51-170)$ & $64(51-131)$ & $82.0(63-170)$ & $124(86-168)$ \\
Weight $(\mathrm{kg})$ & $11.5(4.8-68.6)$ & $5.7(4.8-22)$ & $10.8(5.2-60)$ & $25.6(12.3-68.6)$ \\
Body surface area $\left(\mathrm{m}^{2}\right)$ & $0.53(0.26-1.79)$ & $0.32(0.26-0.89)$ & $0.51(0.3-1.68)$ & $0.94(0.55-1.79)$ \\
Dose area product total $\left(\mu \mathrm{Gym}^{2}\right)$ & $228.7(33.3-7249.7)$ & $45.9(33.3-144.5)$ & $216.2(57.5-7249.7)$ & $649.5(310.9-3102.5)$ \\
Dose area product CT run $(\mu \mathrm{Gym}$ & \\
Total contrast-medium $/ \mathrm{wt}(\mathrm{ml} / \mathrm{kg})$ & $91.8(33-679.3)$ & $45.4(33-144.5)$ & $67.0(48-679.3)$ & $288.0(112.1-655.5)$ \\
CT contrast-medium/wt $(\mathrm{ml} / \mathrm{kg})$ & $4.2(1.3-9.0)$ & $2.7(1.3-4.7)$ & $6.1(2.8-9.0)$ & $3.5(1.8-8.2)$ \\
\hline
\end{tabular}

$\mathrm{CT}=$ computed tomography

frequently seen. ${ }^{6}$ Cardiac catheterisation before total cavopulmonary connection is yet often recommended $^{7,8}$ and it remains essential for interventions.

With the introduction of flat-detector-equipped c-arms in paediatric catheterisation laboratories, there is a novel possibility of gaining flat-detector computed tomography images, mainly as angiographic computed tomography images with a high quality. These angiographic images rendered in a three-dimensional model can be used immediately during the same investigation by displaying it live on a fluoroscopy monitor to guide catheter interventions and to select the most appropriate c-arm angle. ${ }^{9-12}$ The scanning time is at least 5 seconds, and thus pulsatile motion of the heart and great vessels, as well as breathing generate artefacts. Close to the heart, these artefacts are distinctive; however, extracardial structures such as the cavopulmonary connections can be imaged by flat-detector computed tomography in a high quality. ${ }^{9,10}$

The use of flat-detector computed tomography in catheterisation of congenital heart disease is a new imaging procedure and there are only few data about the investigative impact of this technique on cavopulmonary connections. Therefore, we evaluated and report our experience with flat-detector computed tomography as a new imaging modality in 31 consecutive cases with cavopulmonary connections.

\section{Patients and methods}

We analysed all patients with cavopulmonary connection who underwent invasive imaging in our cardiac catheterisation laboratory retrospectively in an 18month period between January, 2010 and June, 2011. In this period, we also investigated three patients without the use of flat-detector computed tomography but excluded these cases from our study.

The most common congenital heart defects in the investigated patients were different kinds of double outlet right ventricle (8 out of 31 cases), double inlet left ventricle (8 out of 31 cases), and hypoplastic left heart syndromes (6 out of 31 cases), followed by pulmonary atresia with intact ventricular septum ( 3 out of 31 cases), tricuspid atresia ( 3 out of 31 cases), and others (3 out of 31 cases). We divided the studied patients in three groups (Table 1): in the first group (eight patients), we investigated all patients after partial cavopulmonary connection soon after operation; in six of these patients, the flat-detector computed tomography was performed on the first post-operative day, in two on the second post-operative day (after surgery), and all patients except one were breathing spontaneously. The administration of the diluted contrast medium was always by hand injection through the pre-existing central venous line in the right jugular vein. There were three patients with a bilateral bidirectional cavopulmonary connection. In two of these patients, contrast was administered additionally through the left external jugular vein and in one patient the left cubital vein was used. The flat-detector computed tomography acquisition by rotational angiography was used exclusively in these investigations.

The second group included 13 patients in whom flat-detector computed tomography was applied before planned total cavopulmonary connection. Routinely, the right jugular vein was used for investigations. Flat-detector computed tomography was performed by contrast dye administration through a pigtail catheter and machine-injection in the superior caval vein. An arterial access was only inserted in cases of anamnestic, clinical, or echocardiographic signs of anomalies. We administered contrast dye in the partial cavopulmonary connection several times and simultaneously in the aorta during the flat-detector computed tomography (3 out of 13 cases) to display the spatial anatomical relationship. Whenever interventional catheterisation was required, the three-dimensional vascular model was used by merging it with the fluoroscopic picture for three-dimensional navigation. 
Table 2. Modified Likert scale concerning the diagnostic utility of flat-detector computed tomography (adapted from ${ }^{13}$ ).

\begin{tabular}{ll}
\hline Misleading & $\begin{array}{c}\text { Misleading information for pre-operative } \\
\text { planning or intervention } \\
\text { Not useful } \\
\text { No useable information for pre-operative } \\
\text { planning or intervention } \\
\text { Useful information for pre-operative planning or } \\
\text { intervention, but only minimally superior to } \\
\text { the already available information of } \\
\text { angiographies } \\
\text { The scan added important information to what } \\
\text { was already available } \\
\text { The surgery or the intervention could not have } \\
\text { Essential } \\
\text { been performed appropriately without the } \\
\text { additional information provided }\end{array}$ \\
\end{tabular}

In the third group, we investigated 10 patients with failing total cavopulmonary connection requiring invasive catheter evaluation or intervention. The flat-detector computed tomography was mostly performed by simultaneous contrast administration through a pigtail catheter in the superior and the inferior caval vein simultaneously. The threedimensional model was used for three-dimensional navigation in case of interventions.

In all three groups, the ability to provide diagnostic information for surgery was jointly determined by one paediatric cardiologist and one paediatric cardiac surgeon in every case. The diagnostic contribution of flat-detector computed tomography was rated using a standardised, previously published 5-point Likert scale, see Table $2 .{ }^{13}$ In the first group, the flat-detector computed tomography was used to gain early postoperative information about the anatomical structure and the function of the cavopulmonary connection. In the second and third groups, the benefit of using overlaid three-dimensional images obtained from flatdetector computed tomography during catheter-based interventions was jointly analysed by the operator and one paediatric cardiologist. Again, a classification of the Likert scale (Table 2) was used.

We used flat-detector computed tomography based on rotational angiography with three-dimensional image reconstruction of a vascular model using volume-rendering technique. If transcatheter intervention was necessary, the three-dimensional model was merged with the fluoroscopic picture for three-dimensional navigation. All examinations were performed on a biplane angiography system equipped with two $20 \times 20$ square centimetre flat panel detectors with a $960 \times 960$ matrix (Axiom Artis, Siemens Medical Solutions, Forchheim, Germany). For image acquisition, the c-arm with the flat detector rotates around the patient in an array of $180^{\circ}$ in addition to the fan angle in 5 seconds; therefore a volume data set is gained by pulsed $\mathrm{X}$-rays with a frame rate of 30 per second and a dose of 0.36 microgray per image. The tube voltage was fixed at 90 kilovolt and tube current was automatically adapted. The anti-scatter grid was removed in patients less than 20 kilograms. Cross-sectional images were rendered with 0.4 millimetre thickness in a $500 \times 500$ matrix format using the kernel-type "HU". Post-processing of the data set was performed on a commercially available workstation (Leonardo DynaCT, InSpace 3D software, Siemens Medical Solutions). The injected contrast agent was Imeron ${ }^{\circledR} 350$ (Iodine 350 milligrams per millilitre; Bracco Imaging Deutschland $\mathrm{GmbH}$; Konstanz, Germany); it was always diluted with saline in a median concentration of $50 \%$ (range 30-75\%).

\section{Results}

During the study period of 18 months, a total of 34 patients with cavopulmonary connection were investigated in our catheterisation laboratory. Flatdetector computed tomography was applied in 31 of these patients and they were included in the study. In 14 patients $(45 \%)$, an interventional procedure was performed. In the other 17 cases (55\%), it was a solely diagnostic catheterisation.

The median age was 1.9 years - range $0.3-42.5$ years, median weight was 11.5 kilograms - range 4.8-68.6 kilograms, and $61 \%$ of the patients were male (Table 1).

We subsumed eight (26\%) patients early postoperatively after surgically performed upper cavopulmonary connection in the first group; in four cases $(50 \%)$, we detected pathologies, which led to therapeutical consequences (Fig 1). In one patient, there was an inverse blood flow in the left pulmonary branch caused by huge aortopulmonary collateral arteries. The patient underwent interventional catheterisation a few days later with occlusion of these arteries. There were two patients with bilateral Glenn anastomosis who had an obstruction of the pulmonary bifurcation between the two upper caval veins. In these cases, we administered an antithrombotic therapy (Fig 2). There was one patient who had a thrombosis of the confluence of the left jugular and the left subclavian vein; thus, a thrombolysis was performed (Fig 3).

In the second group, we included $13(42 \%)$ patients with upper cavopulmonary connection before completion of a total cavopulmonary connection (Fig 4). Of these investigations, seven $(54 \%)$ were interventional catheters. In two cases, we dilated a stenosis of pulmonary branches; in four cases, the aim was an occlusion of aortopulmonary collateral arteries and additionally in one case a balloon dilatation of an aortic coarctation. 


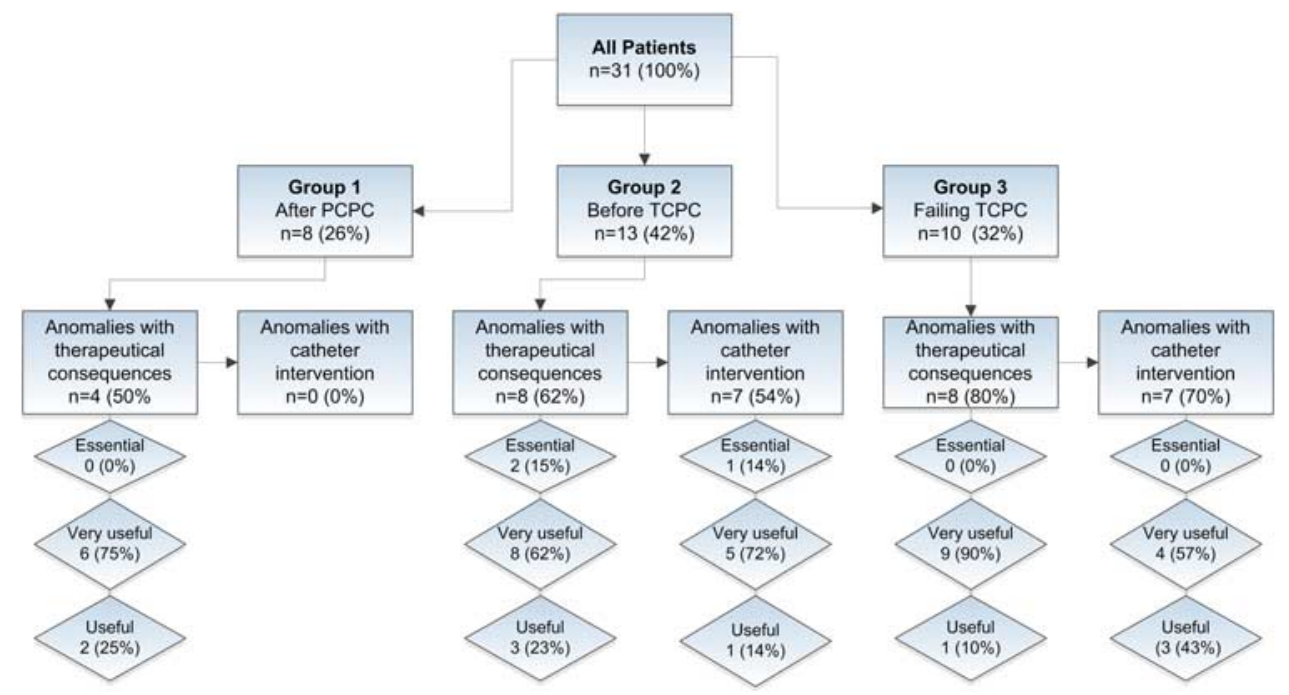

Figure 1.

Results of investigation and rating of the diagnostic utility of flat-detector computed tomography in 31 patients with cavopulmonary connection; number (percentage in the group). PCPC = partial cavopulmonary connection; TCPC = total cavopulmonary connection.
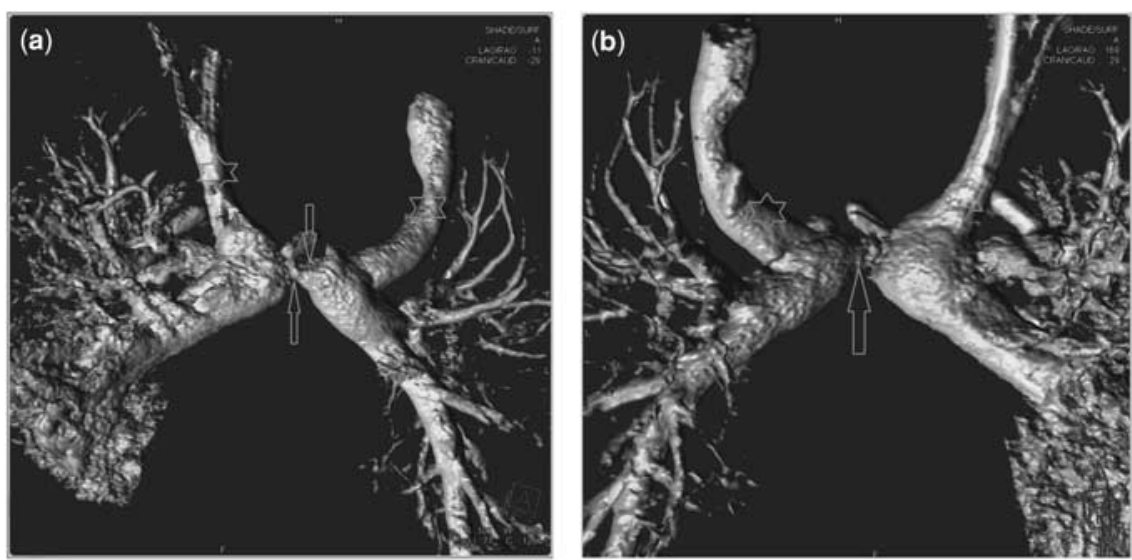

Figure 2 .

Severe stenosis of the bifurcation of the pulmonary artery. A 4-month-old boy with double outlet right ventricle, transposition of the great arteries, and sub-pulmonary stenosis, 1 day after surgery for bilateral, bidirectional Glenn anastomosis. An anticoagulative therapy was performed as consequence of thes residual pathologies. Superior caval veins marked by the stars, stenosis of the pulmonary artery by the arrows; (a) with the view from frontal and inferior and (b) from head and posterior.

In the third group, 10 patients with total cavopulmonary connection $(32 \%)$ were included. Interventions were performed in seven cases $(70 \%)$ : stent placement in pulmonary arteries or the extracardial tunnel (3 out of 7 cases; Figs 5 and 6) and occlusion of aortopulmonary collateral arteries (4 out of 7 cases).

Overall, both flat-detector computed tomography images and overlaid three-dimensional reconstructions were rated superior - "essential", "very useful", or "useful" - to conventional angiography alone in all cases. The diagnostic value flat-detector computed tomography was classified as follows: two (7\%) were rated "essential", $23(74 \%)$ were rated "very useful", six (19\%) were rated "useful", and none was rated "not useful" or "misleading".

In 14 patients in whom interventions were performed, the utility of the merged three-dimensional reconstructions of the flat-detector computed tomography with the real-time fluoroscopic image was rated as follows: One case $(7 \%)$ was rated "essential", $9(64 \%)$ were rated "very useful", 4 $(29 \%)$ were rated "useful", and none was rated "not useful" or "misleading" (Fig 1).

The mean required amount of contrast agent for the rotational angiography was 2.4 plus or minus 1.1 millilitres per kilogram, with a range of $0.7-4.7$ millilitres per kilogram. On average, the percentage 

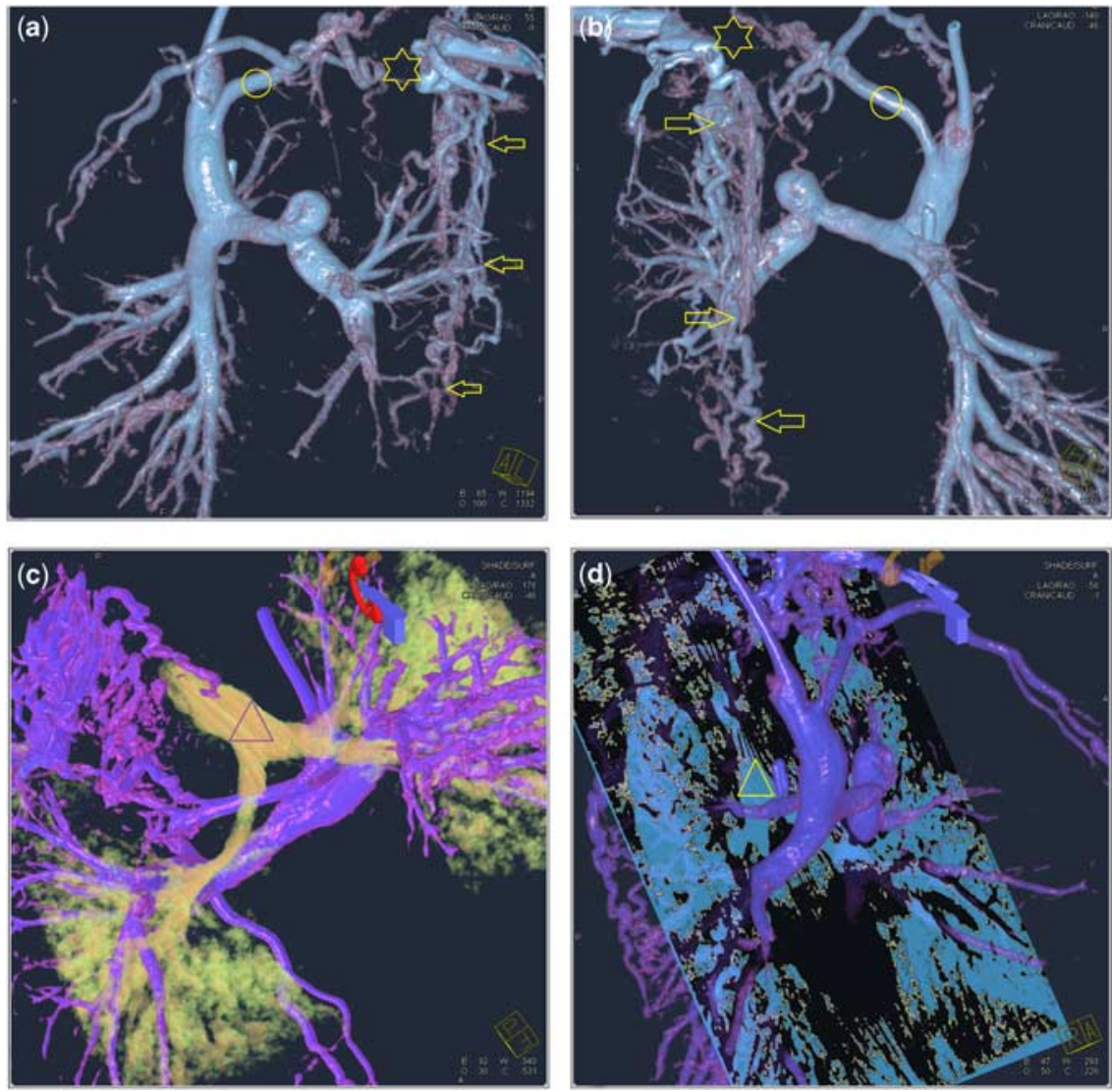

Figure 3.

Thrombosis of the left Pirigoff angle (star). A 4-month-old baby with bypoplastic left heart syndrome 1 day after Glenn anastomosis. Huge paravertebral collateral veins (arrows) and small brachiocephalic vein (circle). Antithrombotic therapy followed. (a) shows the vascular model from anterior and (b) from posterior. In (c), the air passages are also reconstructed to demonstrate the spatial relationships; (d) shows an embedded multi-planar section with the air passages, which may belp for surgical planning.
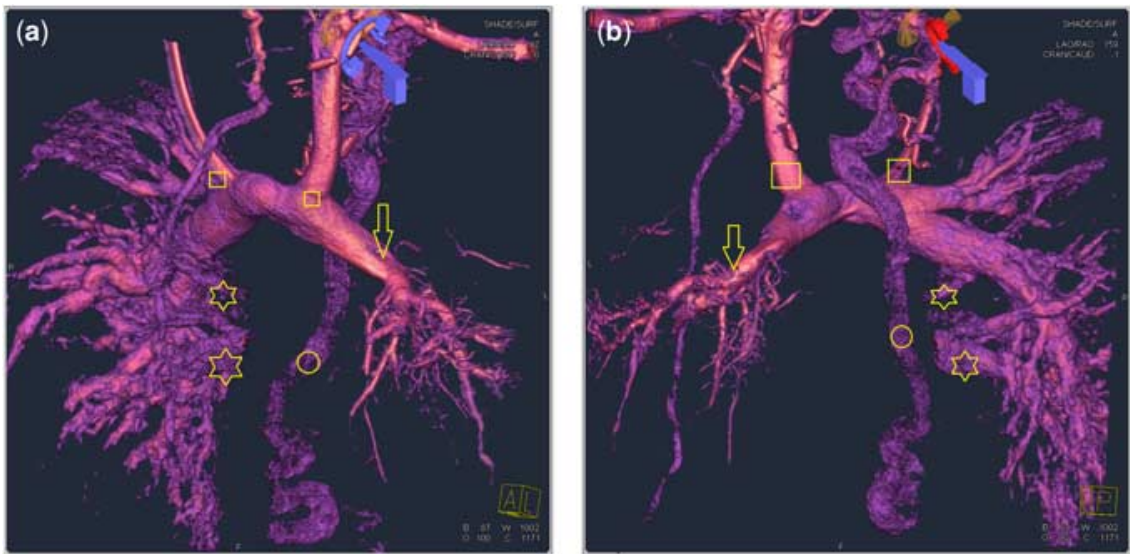

Figure 4 .

Total pulmonary vein occlusion of the left side in a 1-year-old boy with bilateral (quarters) bidirectional Glenn anastomosis with dextrocardia, heterotaxy syndrome, and an unbalanced atrioventricular septal defect. Contrast medium given through the left cubital vein and through a pigtail catheter via the right jugular vein in the left pulmonary artery. Reversal blood flow in the left pulmonary artery (seen in the dynamic rotational angiography) with small peripheral pulmonary arteries (arrow). Pulmonary veins are displayed on the right side (stars), and none on the left side. Collateral blood flow over the azygos vein (circle). (a) is the view from left anterior and (b) from left posterior. 

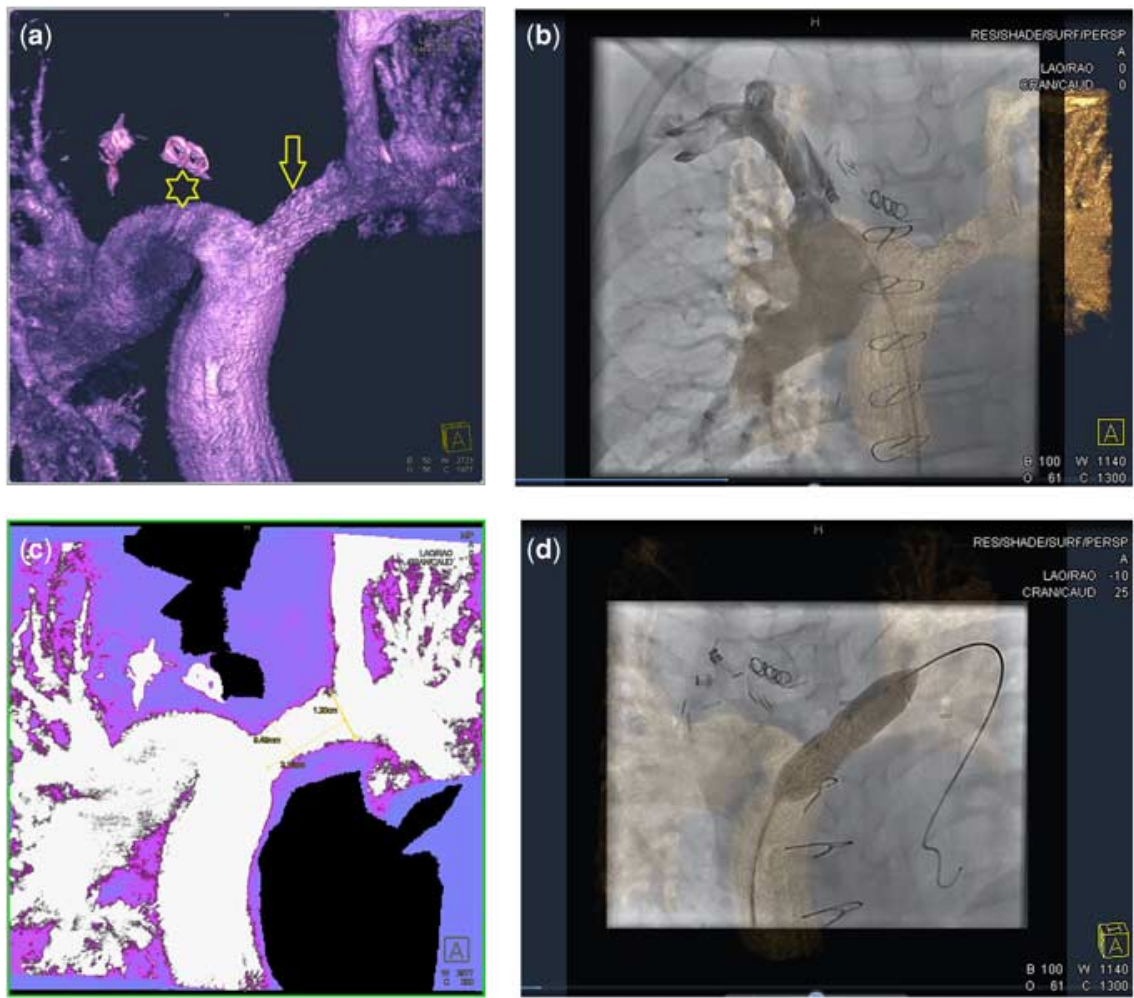

Figure 5.

Total cavopulmonary anastomosis with bilateral, bidirectional Glenn anastomosis. Nine-year-old boy with double inlet left ventricle, 3 years after surgery. Preexisting 10 millimetre stent (arrow) in the left pulmonary artery. Contrast medium given through left cupital vein by handinjection and through a pigtail catheter by machine injection in the extracardial conduit. (a) shows the three-dimensional model; the right superior caval vein is not contrasted and the wash-out effect is seen (star). (b) shows the overlaid three-dimensional model on fluoroscopy and contrast administration in the right superior caval vein. (c) is an imaging in maximum intensity projection for measurements. In (d), the three-dimensional navigation for balloon dilatation of the stent is demonstrated.

of the dose by flat-detector computed tomography of the total investigation was $49 \%$ of the entire contrast used during the procedure (range 25-100\%). The median dose area product of flat-detector computed tomography was 91.8 microgray square metres (range 33.0-679.3 microgray square metres). Flat-detector computed tomography contributed an average of $43 \%$ (range 9-100\%) of the entire radiation dose per procedure. Owing to the fact that tube current was automatically adapted by the X-ray imaging chain, a patient's body surface area is a predictor of the dose area product. ${ }^{14}$

\section{Discussion}

In cavopulmonary connections, residual pathologies may generate severe side effects such as reduced physical capacity, oedema, thrombosis, or protein-losing enteropathy with lymphatic malformation; ${ }^{15-19}$ therefore, an optimal imaging modality is essential. Today, cardiac ultrasound is the standard procedure for pre-operative imaging of congenital heart disease. However, it has a limited sensitivity in detecting great vessel stenosis in cavopulmonary connection., ${ }^{7,18}$ Cardiac catheterisation cannot be performed routinely, but will be indicated if there are remaining questions after echocardiography. This can be caused by bad acoustic windows, difficult spatial relationships of cardiac and vascular structures, and if other imaging modalities such as magnetic resonance imaging or multi-detector row computed tomography are not viable. ${ }^{5,7,8,19}$ Cardiac catheterisation is required for interventions or if an invasive haemodynamic evaluation is necessary.

Recently computed tomography imaging in congenital heart disease has been used increasingly because of improved temporal resolution and detector coverage of multi-detector row computed tomography systems and the development of low-dose imaging algorithms. High image quality can be achieved even in neonates. ${ }^{20-22}$ The main advantage of flat-detector computed tomography imaging is the possibility of performing it during cardiac catheterisation with admission of contrast medium directly in the volume of interest, which makes it easily distinguishable from the surrounding tissue and therefore allows 

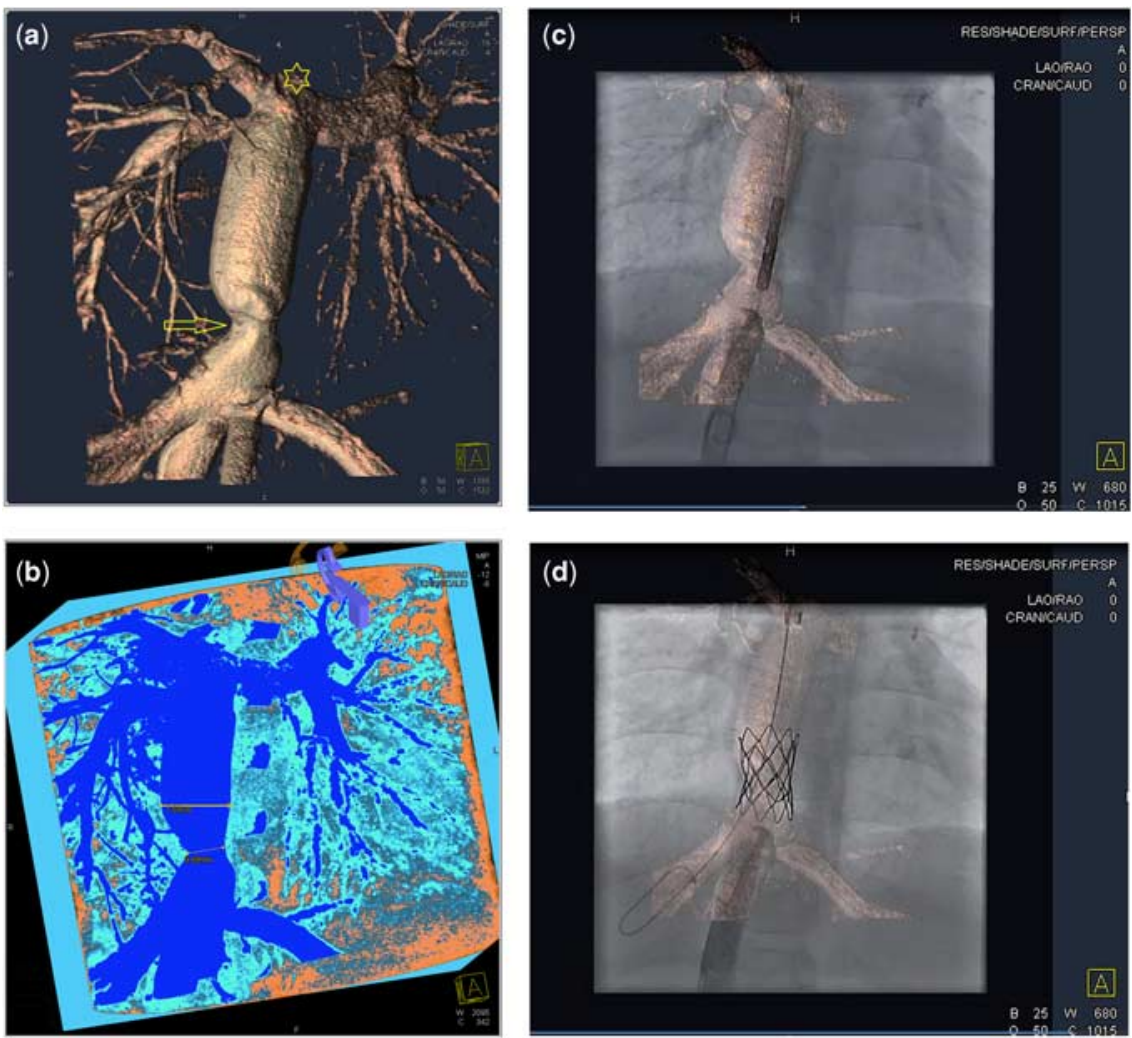

Figure 6 .

Severe stenosis of the anastomosis between inferior caval vein and the conduit (arrow). An 11-year-old boy with bypoplastic left heart syndrome, total cavopulmonary connection, and extracardial conduit. Stent implantation in the stenosis. (a) shows the three-dimensional model in volume-rendering technique. Contrast admission over a pigtail catheter in the conduit. Owing to wash out, the superior caval vein is not contrasted (star). (b) is the same data set in major intensity projection for measurements. (c) is the overlaid three-dimensional model on fluoroscopy during the implantation procedure of a stent. Position of the stenosis and the liver veins are always displayed and threedimensional model follows every new angulation of the c-arm. (d) shows the inflated stent.

fast three-dimensional reconstructions. Thus, these vascular models are immediately available during the invasive investigation in the catheterisation laboratory extending hitherto available imaging modalities. Not least, in the hands of paediatric cardiologists this means an easy and fast access to a modern cross-sectional imaging technique for this profession.

In a series of 31 patients, we found that flat-detector computed tomography used as angiographic computed tomography in cavopulmonary connections provides valuable additional information, both for diagnostic purposes and for the use in interventions to conventional biplane angiographies in all of our cases. We rated the impact of flat-detector computed tomography much higher compared with other publications $(70-90 \%) ;, 10$ however, compared with other anatomic regions, the cavopulmonary connections are visualised much better by this technique because of less movement artefacts and a slow blood flow. With this advanced imaging technique and an adjusted application protocol, there is an improved diagnostic accuracy in cavopulmonary connections. At the same time, the pulmonary veins can be visualised entirely. After creation of a three-dimensional vascular model, this can be viewed in any plane, enabling a demonstration in a "surgical angle of view", and can be rotated in any desired position. Moreover, extracardial structures such as air passages, oesophagus, or bones can be displayed in addition to the vascular model, and their spatial relationship can be demonstrated. Breathing artefacts can be avoided by breath-holding; however, we investigated nearly all patients breathing normally. Breathing artefacts were generally not disturbing. The adverse effect of pulsation on imaging is most considerable in the arteries close to the heart. This can be minimised by reduction of the heart-stroke volume, for example, by rapid heart pacing, but extracardial structures such as the cavopulmonary connections can be visualised accurately under normal cardiac actions and without electrocardiogram triggering. Measurement of vascular diameters, for example before stent implantation, should not be performed in the three-dimensional model, because the result differs from the segmented data, the chosen colour, opacity, 
and lighting settings. In all cases, we were able to perform reliable sizing in rotational angiographies before any reconstruction and also in two-dimensional multi-planar reconstructions or in two-dimensional maximum intensity projections (see Figs $5 \mathrm{C}$ and $6 \mathrm{~B}$ ).

In the eight patients who underwent the partial cavopulmonary connection procedure, we detected residual anomalies in four patients $(50 \%)$ by an early post-operative imaging by flat-detector computed tomography with creation of a high-resolution threedimensional vascular model of the cavopulmonary connection. In all of the four cases, the pathological findings led to therapeutic consequences. The imaging of the partial cavopulmonary connection does not need a new vascular access, because the pre-existing central venous catheter can be used for contrast administration.

Overall, we used the new technique of flat-detector computed tomography in the 18 -month period in $25 \%$ of all cardiac catheterisations of congenital heart disease successfully and without adverse effects. We mainly investigated extra-cardiac regions and used flat-detector computed tomography in questions concerning the aorta, pulmonary artery, and the cavopulmonary connections. Whenever there were questions easy to answer by a single conventional angiography, we did not apply flat-detector computed tomography.

Flat-detector computed tomography in the context of invasive imaging of congenital heart disease is new. Therefore, we have to examine the radiation exposure and the amount of the contrast material used in these cases. The radiation dose used is displayed by the dose area product. The body surface area is a predictor for the dose area product because of the automatic exposure control of flat-detector computed tomography. ${ }^{14}$ The effective dose can only be measured in phantoms. For dose assessment in conventional computed tomography, the conventional computed tomography dose index is used; ${ }^{23}$ however, it is not practical because of the extended irritation fields in craniocaudal direction (z-direction) of the big scanners. $^{24,25}$ Thermoluminescence dosimetry measurement is complex and time consuming, and therefore its use is limited. ${ }^{25}$ Dose calculations using Monte Carlo calculations provide accurate results. ${ }^{12,25}$ Real dose measurements utilising acquisition programmes comparable with this type of flat-detector are rare; with an analogous acquisition programme like ours, the radiation exposure seems to be comparable to a 5-second biplane angiography. ${ }^{9}$ The flat-detector computed tomography replaced all angiographies in some cases, and therefore the percentaged radiation dose of the flat-detector computed tomography in these investigations added up to $100 \%$. The median amount of the used contrast medium of a single rotational angiography was 2.4 millilitres per kilogram.
This is more than what we usually need for a conventional angiography but not twice as much. The three-dimensional model allows unlimited views at any desired angle, whereas conventional biplane angiography requires repeated contrast injections in different $\mathrm{c}$-arm positions to evaluate tortuous vascular structures entirely. In these cases, the total amount of contrast is reduced by the use of this new imaging technique. We received good images and diagnostically viable results in cavopulmonary connections even by contrast admission via a central venous line. By contrast enhancement through a peripheral venous line, sufficient imaging was achieved to visualise the venous return to an upper cavopulmonary anastomosis, but we did not examine the impact of peripheral contrast admission systematically. Concerning the long scanning time, the temporal resolution is significantly inferior to the multi-detector row computed tomography. ${ }^{12}$ Therefore, moving artefacts from heart and great vessels, as well as breathing, can cause interferences, which are most evident close to the heart. ${ }^{10}$ Therefore, we do not use rotational angiography to visualise intracardial structures, because in this region additional procedures such as rapid pacing are necessary to reduce moving artefacts. Especially in cavopulmonary connections, a high-resolution imaging quality by flat-detector computed tomography can be achieved because of minimal pulsatile movement artefacts. The diagnostic value of a rotational angiography is rated above a conventional angiography in all cases and replaces repeating conventional angiographies in different angulations. Flat-detector computed tomography as an innovative and advanced imaging modality improves the diagnostic accuracy in the catheterisation of congenital heart disease and is valuable in catheter-based interventions.

\section{Limitations}

The study is solely retrospective; we included almost all patients with cavopulmonary connection and did not assign them randomly to imaging strategies with and without the use of flat-detector computed tomography. The rating of the image quality and the use in interventions by the paediatric cardiologists and the surgeon cannot be solely objective. However, best efforts to avoid bias included the use of a standardised score and the consensus of two experienced investigators in all cases.

\section{Conclusion}

Flat-detector computed tomography in cavopulmonary connections provides accurate diagnostic information by a single injection of contrast dye 
exceeding conventional biplane angiographies. This advanced technique can be used easily and safely during catheterisation of congenital heart disease, but also as direct post-operative three-dimensional imaging modality after surgery for partial cavopulmonary connection. It is not associated with unreasonably added radiation or contrast exposure. According to our experience, in 31 patients with cavopulmonary connections, the main advantages of this new imaging modality include the unlimited views on the high-resolution three-dimensional vascular models for surgical planning, the use of three-dimensional models in interventions, and the easy access to this cross-sectional modality for paediatric cardiologists.

Flat-detector computed tomography imaging in the paediatric cardiac catheterisation laboratory is still new, and further preferably randomised studies on a larger scale are needed to define the optimal role of this imaging modality.

\section{References}

1. Girod DA, Fontan F, Deville C, Ottenkamp J, Choussat A. Longterm results after the Fontan operation for tricuspid atresia. Circulation 1987; 75: 605-610.

2. Nakata S, Imai Y, Takanashi Y, et al. A new method for the quantitative standardization of cross-sectional areas of the pulmonary arteries in congenital heart diseases with decreased pulmonary blood flow. J Thorac Cardiovasc Surg 1984; 88: 610-619.

3. Senzaki H, Isoda T, Ishizawa A, Hishi T. Reconsideration of criteria for the Fontan operation. Influence of pulmonary artery size on postoperative hemodynamics of the Fontan operation. Circulation 1994; 89: 1196-1202.

4. Harris MA, Cosulich MT, Gillespie MJ, et al. Pre-Fontan cardiac magnetic resonance predicts post-Fontan length of stay and avoids ionizing radiation. J Thorac Cardiovasc Surg 2009; 138: 941-947.

5. Brown DW, Gauvreau K, Moran AM, et al. Clinical outcomes and utility of cardiac catheterization prior to superior cavopulmonary anastomosis. J Thorac Cardiovasc Surg 2003; 126: 272-281.

6. Holzer RJ, Sisk M, Chisolm JL, et al. Completion angiography after cardiac surgery for congenital heart disease: complementing the intraoperative imaging modalities. Pediatr Cardiol 2009; 30: 1075-1082.

7. Ba HO, Marini D, Kammache I, et al. Preoperative evaluation of candidates for total cavopulmonary connection: the role of echocardiography and cardiac catheterization. Arch Cardiovasc Dis 2009; 102: 303-309.

8. Ro PS, Rychik J, Cohen MS, Mahle WT, Rome JJ. Diagnostic assessment before Fontan operation in patients with bidirectional cavopulmonary anastomosis: are noninvasive methods sufficient? J Am Coll Cardiol 2004; 44: 184-187.
9. Glatz AC, Zhu X, Gillespie MJ, Hanna BD, Rome JJ. Use of angiographic $\mathrm{CT}$ imaging in the cardiac catheterization laboratory for congenital heart disease. JACC Cardiovasc Imaging 2010; 3: 1149-1157.

10. Glockler M, Koch A, Greim V, et al. The value of flat-detector computed tomography during catheterisation of congenital heart disease. Eur Radiol 2011; 21: 2511-2520.

11. Gupta R, Cheung AC, Bartling SH, et al. Flat-panel volume CT: fundamental principles, technology, and applications. Radiographics 2008; 28: 2009-2022.

12. Kalender WA, Kyriakou Y. Flat-detector computed tomography (FD-CT). Eur Radiol 2007; 17: 2767-2779.

13. Ellis AR, Mulvihill D, Bradley SM, Hlavacek AM. Utility of computed tomographic angiography in the pre-operative planning for initial and repeat congenital cardiovascular surgery. Cardiol Young 2010; 20: 262-268.

14. Wielandts JY, Smans K, Ector J, De Buck S, Heidbuchel H, Bosmans H. Effective dose analysis of three-dimensional rotational angiography during catheter ablation procedures. Phys Med Biol 2010; 55: 563-579.

15. Feldt RH, Driscoll DJ, Offord KP, et al. Protein-losing enteropathy after the Fontan operation. J Thorac Cardiovasc Surg 1996; 112: 672-680.

16. Glockler M, Severin T, Arnold R, et al. First description of three patients with multifocal lymphangiomatosis and protein-losing enteropathy following palliation of complex congenital heart disease with total cavo-pulmonary connection. Pediatr Cardiol 2008; 29: 771-774.

17. Rychik J. Management of protein-losing enteropathy after the Fontan procedure. Semin Thorac Cardiovasc Surg Pediatr Card Surg Annu 1998; 1: 15-22.

18. Fraisse A, Colan SD, Jonas RA, Gauvreau K, Geva T. Accuracy of echocardiography for detection of aortic arch obstruction after stage I Norwood procedure. Am Heart J 1998; 135: 230-236.

19. McMahon CJ, Eidem BW, Bezold LI, et al. Is cardiac catheterization a prerequisite in all patients undergoing bidirectional cavopulmonary anastomosis? J Am Soc Echocardiogr 2003; 16: 1068-1072.

20. Achenbach S, Marwan M, Ropers D, et al. Coronary computed tomography angiography with a consistent dose below $1 \mathrm{mSv}$ using prospectively electrocardiogram-triggered high-pitch spiral acquisition. Eur Heart J 2010; 31: 340-346.

21. Lell M, Hinkmann F, Anders K, et al. High-pitch electrocardiogram-triggered computed tomography of the chest: initial results. Invest Radiol 2009; 44: 728-733.

22. Lell MM, May M, Deak P, et al. High-pitch spiral computed tomography: effect on image quality and radiation dose in pediatric chest computed tomography. Invest Radiol 2011; 46: 116-123.

23. Shope TB, Gagne RM, Johnson GC. A method for describing the doses delivered by transmission X-ray computed tomography. Med Phys 1981; 8: 488-495.

24. Fahrig R, Dixon R, Payne T, Morin RL, Ganguly A, Strobel N. Dose and image quality for a cone-beam C-arm CT system. Med Phys 2006; 33: 4541-4550.

25. Kyriakou Y, Deak P, Langner O, Kalender WA. Concepts for dose determination in flat-detector CT. Phys Med Biol 2008; 53: 3551-3566. 